\title{
Spinocerebellar ataxia type 40
}

INSERM

\section{Source}

INSERM. (1999). Orphanet: an online rare disease and orphan drug data base.

Spinocerebellar ataxia type 40. ORPHA:423275

Spinocerebellar ataxia type 40 (SCA40) is a very rare subtype of autosomal dominant cerebellar ataxia type 1, characterized by the adult-onset of unsteady gait and dysarthria, followed by wide-based gait, gait ataxia, ocular dysmetria, intention tremor, scanning speech, hyperreflexia and dysdiadochokinesis. 